\title{
Selection of Hadronic W-decays in DELPHI with Feed Forward Neural Networks - an Update
}

\author{
K.-H. Becks ${ }^{\text {a }}$,J. Drees ${ }^{\text {a }}$, U. Müller*a and H. Wahlen ${ }^{\text {a }}$ \\ aBergische Universität, Fachbereich Physik, Gaußstr. 20, D-42097 Wuppertal, Germany
}

Since 1998 feed forward neural networks have been successfully applied to select candidates of hadronic Wdecays measured at different center of mass-energies by the DELPHI collaboration at the Large Electron Positron collider at CERN. To prepare the final publication, the neural network was adapted to all center of mass-energies. Detailed studies were performed concerning the level of preselection, the choice of network parameters and especially of the network architecture. The number of hidden nodes was optimized by testing different pruning methods. All studies and results will be discussed.

\section{Introduction}

From 1996 to 2000 the Large Electron Positron collider (LEP) at $\mathrm{CERN}^{2}$ ran above the threshold energy for W-pair-production $e^{+} e^{-} \rightarrow W^{+} W^{-}$ at center of mass-energies from 161 to $209 \mathrm{GeV}$. The determination of the $\mathrm{W}$-production crosssection and the direct measurement of the mass of the W-boson allow new tests of the standard model.

In 1998 the first analysis started using a feed forward neural network to separate hadronic WW-decays $W^{+} W^{-} \rightarrow q \bar{q} q \bar{q}$ measured by the DELPHI $^{3}$ detector at $189 \mathrm{GeV}$ from different backgrounds, mainly from QCD-events $e^{+} e^{-} \rightarrow$ $Z^{0} / \gamma^{*} \rightarrow q \bar{q}(g g)$ and from hadronic decays of the Z-pair-production $e^{+} e^{-} \rightarrow Z Z \rightarrow q \bar{q} q \bar{q}$. Due to a clear improvement in the selection quality the neural network analysis was chosen for the official DELPHI cross-section analysis in the hadronic decay channel. Therefore the network had to be adapted to all other center of mass-energies.

\section{Feed Forward Neural Network}

The first feed forward network was based upon the JETnET package [1] and consisted of a simple 13-7-1 architecture. The input variables were

\footnotetext{
*corresponding author, mueller@whep.uni-wuppertal.de ${ }^{2}$ European Organization for Nuclear Research

${ }^{3}$ Detector with Lepton, Photon and Hadron Identification
}

chosen from the previous DELPHI standard or other high-energy physics analyses and a study with self-organizing maps [2].

The selection quality, indicated by the product of signal efficiency and purity, could be increased by about $10 \%$. A more detailed overview of this first analysis and a comparison between the results from the neural network and from the previous analysis using linear cuts can be found in a DELPHI publication [3] and in the proceedings of ACAT2000 [4].

\section{Optimization of the Neural Network}

For the final publication the analyses at all energies from 183 to $207 \mathrm{GeV}$ have to be consistent and to give the best possible separation between signal and background. After an energy dependent preselection the same neural network concerning the architecture and the internal parameter settings should be used. Only the trainings are performed separately at each energy.

\subsection{Preselection}

If the preselection is too tight, the signal efficiency is too small to reach a top performance. If the preselection is very loose, the network concentrates on clear discrepancies between signal and background and doesn't take into account subtile differences. This results in a worse performance. But the selection quality is very stable varying 
the preselection around the working point.

\subsection{Parameters and Configuration}

Different numbers of training events and different training samples were used as well as a wide range of the learning rate $\eta\left(0.0025_{-0.0015}^{+0.015}\right.$ with and without decrease during the training) and the momentum term $\alpha(0.56 \pm 0.3)$. In all cases the selection results were compatible within the statistical uncertainties.

\subsection{Pruning}

The first architecture 13-7-1 had been selected by trial-and-error. Now two pruning procedures were tested to check this choice.

In the first procedure, implemented in JETNET, a complexity term was added to the fitness error $E: E \rightarrow E+\lambda \sum_{i j} \frac{\omega_{i j}^{2} / \omega_{0}^{2}}{1+\omega_{i j}^{2} / \omega_{0}^{2}}$. The parameters $\lambda$ and $\omega_{0}^{2}$ are also used for updating the weights $\omega_{i j}$ whereas an additional parameter $D$ is a threshold for the procedure and specifies how much pruning is to be performed. During the training the number of hidden nodes was reduced depending on the value of $D$. When starting with more than 4 nodes the number was reduced to 4,4 were reduced to 3 .

The second procedure was pruning-by-eye. After an interval of cycles all weights below a threshold were set to zero and then the training was continued. In all tests with different intervals and different thresholds no node, neither a hidden nor an input one, was completely disconnected and thus found to be negligible.

All studies with both procedures gave a much better selection quality than the previous cut analysis. But although less than 7 hidden nodes seem to be enough no significant improvement compared to the basic network was found.

\section{Systematic Error and Final Results}

The variations of the network parameters and configurations were found to be negligible. In all further studies the neural network was used like a mathematical function, i.e. the trainings at each energy were fixed. Different techniques, well known from the previous or other high-energy physics analyses, were used:
- comparison of different simulated event samples,

- comparison of real data and simulated events,

- taking into account detector resolution.

The cross-section is determined from a binned maximum likelihood fit to the output distribution of the neural network taking into account the expected background in each bin. The fit also gives the systematic error using the uncertainties of efficiency and background and other analysisindependent errors. The result for $189 \mathrm{GeV}$ is: $\sigma_{W^{+} W^{-} \rightarrow q \bar{q} q \bar{q}}=7.36 \pm 0.26$ (stat) \pm 0.10 (syst) $p b$. The result of the previous standard analysis $(7.56 \pm 0.28($ stat $))$ is in agreement within the uncertainties. The systematic error of the cut analysis is expected from the previous years to be compatible but the statistical error is somewhat higher due to the worse selection quality.

\section{Summary}

In the DELPHI collaboration a feed forward neural network is successfully applied for the determination of the hadronic WW-cross-section. The network which clearly outperforms the standard cut analysis was adapted to other center of mass-energies. Different studies concerning the choice of network parameters and settings were performed and the neural network was used as a mathematical function to determine the systematical error.

\section{REFERENCES}

1. Lønnblad,L., Peterson,C., Pi,H. and Røgnvaldsson, T., JeTnet 3.1 - A Neural Network program for jet discrimination and other High Energy Physics triggering situations Department of Theoretical Physics, University of Lund, Sweden (1994).

2. K.-H. Becks, J. Drees, U. Flagmeyer, U. Müller, NIM A 426, (1999) 599.

3. DELPHI Collaboration, Physics Letters $B$ 479, (2000) 89.

4. K.-H. Becks, P. Buschmann, J. Drees, U. Müller, AIP Conf. Proc. 583, (2001) 80. 